\title{
Protective effect of vitexin reduces sevoflurane-induced neuronal apoptosis through HIF-1 $\alpha$, VEGF and p38 MAPK signaling pathway in vitro and in newborn rats
}

\author{
ZHIPAI LYU ${ }^{1}$, JING CAO ${ }^{2}$, JU WANG ${ }^{1}$ and HONGMEI LIAN ${ }^{1}$ \\ Departments of ${ }^{1}$ Anesthesia and ${ }^{2}$ Pathology, The Third Affiliated Hospital of Zhengzhou University, \\ Henan, Zhengzhou 450052, P.R. China
}

Received January 27, 2016; Accepted October 26, 2017

DOI: $10.3892 / \mathrm{etm} .2018 .5758$

\begin{abstract}
Previous studies have demonstrated that Vitexin possesses antihypertensive, anti-inflammatory and potential anticancer effects. The present study aimed to investigate whether the protective effect of vitexin protects against sevoflurane-induced neuronal apoptosis and the underlying mechanisms of this protective effect. The results demonstrated that Vitexin pretreatment significantly reduced neuronal apoptosis, and inhibited caspase-3 activity, apoptosis regulator BAX protein expression and malondialdehyde levels in sevoflurane-induced newborn rats. In addition, Vitexin pretreatment increased superoxide dismutase and glutathione peroxidase activity. Furthermore, it was revealed that treatment with vitexin induced hypoxia inducible factor $1 \alpha$ subunit (HIF-1 $\alpha)$ and vascular endothelial growth factor (VEGF) protein expression, and suppressed phosphorylated-p38 MAP kinase (p38) protein expression in sevoflurane-induced newborn rat. Together, the results of the current study suggest that the protective effect of vitexin reduces sevoflurane-induced neuronal apoptosis through HIF-1 $\alpha$-, VEGF- and p38-associated signaling pathways in newborn rats.
\end{abstract}

\section{Introduction}

Sevoflurane is a commonly used general anesthetic, which has been widely used in the anesthesia of infants and young children (1). Long-term exposure to sevoflurane anesthesia can cause neurological dysfunction, especially in infants, which can lead to developmental neurodegeneration (1). It may also cause the central nervous system degradation of infants, affecting memory and cognitive function (2). Recent studies have shown

Correspondence to: Mr. Jing Cao, Department of Pathology, The Third Affiliated Hospital of Zhengzhou University, 7 Kangfu Former Street, Henan, Zhengzhou 450052, P.R. China

E-mail: ocsdkgz562772@126.com

Key words: vitexin, sevoflurane, neuronal apoptosis, HIF-1 $\alpha$, VEGF, p38 MAPK that the exposure to clinically relevant doses of narcotic drugs, such as isoflurane and sevoflurane, leads to neurological disorders in rats and mice $(3,4)$. All these results indicate that anesthetic exposure in the early postpartum period may cause permanent damage to the developing brain (3).

Hypoxia inducible factor-1 (HIF-1) is an oxygen-sensitive transcriptional regulator that regulates more than 70 downstream target genes, including vascular endothelial growth factor, erythropoietin, glucose transporter and so on (5). Upregulation of HIF-1 expression may play a neuroprotective role in animal models of focal cerebral ischemia (6).

MAPKs are short for the mitogen-activated protein kinase family. The family members are a group of evolutionarily conserved enzymes in mammalian cells, including four subclasses: ERK1/2, JNK, p38 and ERK5 (7). In recent years, the MAPK family has been found to be an important signal-regulating enzyme between cell surface receptors and determinants of gene expression (8). Therefore, the MAPK family governs almost all the physiological functions and processes such as cell adaptation, proliferation, differentiation, survival, and programmed cell death. And it is the hub between the cytoplasm and the nucleus (8).

VEGF is purified from bovine pituitary follicular cells in 1959 and is a vascular endothelial cell-specific mitogen that promotes the growth of vascular endothelial cells. It lifts the mRNA levels of plasminogen activator (PA) and plasminogen activator inhibitor (PAI) to play a role of activation and regulation on PA and PAI-1, thus enhancing the activity of plasminogen activator and promoting the formation of new capillaries. In addition, the addition of VEGF to the culture medium can increase the survival rate of neurons in the cerebral cortex as well as the number and length of ganglion cell processes, to promote nerve growth.

Vitexin is mainly found in the Crataeguspinnatifida, as one of the main active components of the total flavonoids extracted from the dried fruits (9). In addition, they are also widely found in other plants, such as Trollius, Cajan leaves, willow leaves, short bamboo leaves and Humulus (9). Vitexin has anti-tumor, anti-oxidation, anti-virus, anti-inflammatory, anti-bacterial, antihypertensive and antispasmodic and other biological activities (10). In recent years, it has been reported that vitexin has anti-inflammatory effect (10). The present 
study was designed to investigate whether the protective effect of vitexin protects against sevoflurane-induced neuronal apoptosis and the mechanisms of this protective effect.

\section{Materials and methods}

Cell culture and experimental protocols. Human neuroglioma cells $\mathrm{H} 4$ cell was purchased from Shanghai Cell Bank and cultured in Dulbecco's modified Eagle's medium (DMEM) (Gibco, Grand Island, NY, USA) containing $10 \%$ heat-inactivated fetal bovine serum (Gibco) at $37^{\circ} \mathrm{C}$ with $5 \% \mathrm{CO}_{2}$ in a humidified incubator. Cells were gassed with $0 \%$ (Control group), $4.1 \%$ sevoflurane (Model) and $4.1 \%$ sevoflurane $+100 \mu \mathrm{M}$ vitexin (Vitexin). In Model group, $\mathrm{H} 4$ cell was gassed with $4.1 \%$ sevoflurane for $15 \mathrm{~min}$. Then, $\mathrm{H} 4$ cell was gassed with $4.1 \%$ sevoflurane for $15 \mathrm{~min}$, and then treated with $100 \mu \mathrm{M}$ vitexin for $24 \mathrm{~h}$.

Methyl-thiazolyl-tetrazolium (MTT) assay. After vitexin treatment, $\mathrm{H} 4$ cell was added with MTT $(10 \mu 1,5 \mathrm{mg} / \mathrm{ml})$ for $4 \mathrm{~h}$ at $37^{\circ} \mathrm{C}$. Medium was removed and DMSO sassy was added for $20 \mathrm{~min}$ at $37^{\circ} \mathrm{C}$. The absorbance was measured by a microplate reader at $490 \mathrm{~nm}$ using ELISA reader $\left(\mathrm{Epoch}^{\mathrm{TM}}\right.$; BioTek Instruments, Inc., Winooski, VT, USA).

Sevoflurane exposure. Male SD rats weighing (20 g, 1-2 weeks) and SD rat were maintained at $22 \pm 2^{\circ} \mathrm{C}, 55-5 \%$ humidity, at $12 \mathrm{~h} \mathrm{light/dark} \mathrm{cycle,} \mathrm{and} \mathrm{ad} \mathrm{libitum} \mathrm{access} \mathrm{to} \mathrm{food} \mathrm{and} \mathrm{water.}$ Thirty rats were randomly and equally assigned into three groups: control group, Sevoflurane model group, Sevoflurane model + Vitexin group. Sevoflurane-induced rat was placed into a chamber and exposed to $2.1 \%$ sevoflurane for $2 \mathrm{~h}$. Then, rats of Sevoflurane model + Vitexin group was treated with $50 \mathrm{mg} / \mathrm{kg}$ of Vitexin for 4 weeks.

Assessment of oxidative stress and caspase-3 markers. Total protein was extracted from cell or tissue samples using RIPA sassy (Sigma, St. Louis, MO, USA) and total protein concentration was analyzed with a BCA kit (Sigma). Equal amounts of the proteins $(5 \mu \mathrm{g})$ was incubated with malondialdehyde (MDA) level, and increased superoxide dismutase (SOD) and glutathione peroxidase (GSH-px) activity ELISA Kits or caspase-3 activity ELISA kit. The absorbance was measured by a microplate reader at 450 or $405 \mathrm{~nm}$ using ELISA reader $\left(\right.$ Epoch $^{\mathrm{TM}}$; BioTek Instruments, Inc.).

Western blot analysis. Total protein was extracted from cell or hippocampus tissue samples using RIPA sassy (Sigma) and total protein concentration was analyzed with a BCA kit (Sigma). Equal amounts of the proteins $(50 \mu \mathrm{g})$ were subjected to $6-12 \%$ SDS-PAGE and then transferred to a $0.2 \mu \mathrm{m}$ polyvinylidene difluoride membrane (EMD Millipore, Billerica, MA, USA). The membrane was blocked with 5\% non-fat milk in TBST, and immunobloted overnight using primary antibodies against Bax (Cell Signaling Technology, Inc., Danvers, MA, USA), HIF-1 $\alpha$ (Cell Signaling Technology, Inc.), VEGF (Cell Signaling Technology, Inc.), phosphorylation-p38 MAPK (p-p38 MAPK, Cell Signaling Technology, Inc.) and GAPDH (Cell Signaling Technology, Inc.) overnight at $4^{\circ} \mathrm{C}$. Subsequently, the membranes were washed thrice with

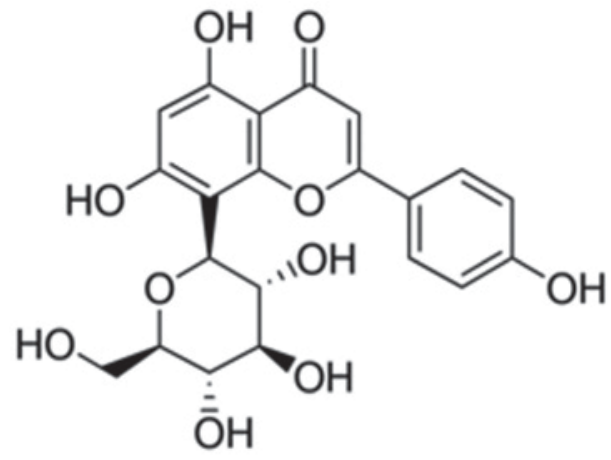

Figure 1. The chemical structure of Vitexin.

TBST and incubated with HRP-linked anti-mouse IgG (Cell Signaling Technology, Inc.) for $2 \mathrm{~h}$ at room temperature and observed using an enhanced chemiluminescence kit (GE Healthcare Life Sciences, Chalfont, UK). Image Lab 3.0 software (Bio-Rad Laboratories, Hercules, CA, USA) was measured Band intensities.

Statistical analysis. Data from each group were expressed as mean \pm standard error. Data were analyzed using one-way analysis of variance (ANOVA). $\mathrm{P}<0.05$ was considered to indicate a statistically significant difference.

\section{Results}

The effects of vitexin on caspase-3 activity and Bax protein expression of sevoflurane-induced newborn rat. The chemical structure of Vitexin was showed at Fig. 1. We firstly used sevoflurane-induced newborn rat treated by vitexin to evaluate the effect of vitexin on nerve apoptosis of sevoflurane-induced newborn rat. we analyzed caspase- 3 activity and Bax protein expression of sevoflurane-induced newborn rat. As shown in Fig. 2, there were significant increases of caspase-3 activity and Bax protein expression in sevoflurane-induced newborn rat, compared with control group. Treatment with Vitexin significantly inhibited sevoflurane-induced caspase-3 activity and Bax protein expression in sevoflurane-induced newborn rat (Fig. 2).

The effects of vitexin on MDA, SOD and GSH-px activities of sevoflurane-induced newborn rat. Next, we examined the anti-oxidative effects of Vitexin in sevoflurane-induced newborn rat. Fig. 3 showed that the increase of MDA level and inhibition of SOD and GSH-px levels in sevoflurane-induced newborn rat were significantly observed, compared with control group. Vitexin treatment with significantly inhibited MDA level and increased SOD and GSH-px levels in sevoflurane-induced newborn rat (Fig. 3).

The effects of vitexin on HIF-1 $\alpha, V E G F$ and p38 MAPK protein expression of sevoflurane-induced newborn rat. Thus, in the current study, we investigated the possible mechanism of Vitexin on nerve apoptosis of sevoflurane-induced newborn rat. The protein of HIF-1 $\alpha$, VEGF and p38 MAPK expression in sevoflurane-induced newborn rat was very higher than those of control group (Fig. 4). Treatment with Vitexin significantly 

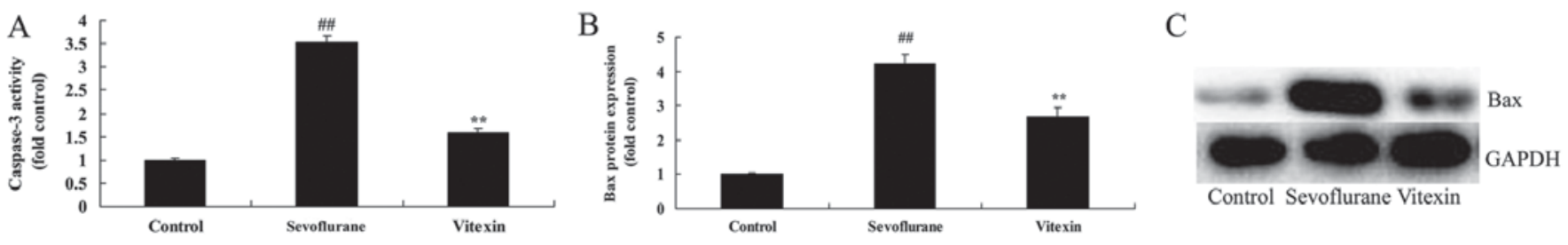

Figure 2. The effects of Vitexin on caspase-3 activity and Bax protein expression of sevoflurane-induced newborn rat. The effects of Vitexin on (A) caspase-3 activity and (B) Bax protein expression and (C) statistical analysis of sevoflurane-induced newborn rat. Control, control group; Sevoflurane, sevoflurane model group; Vitexin, Vitexin + sevoflurane model group. ${ }^{* *} \mathrm{P}<0.01$ compared with the sevoflurane model group; ${ }^{\# \#} \mathrm{P}<0.01$ compared with the control group.
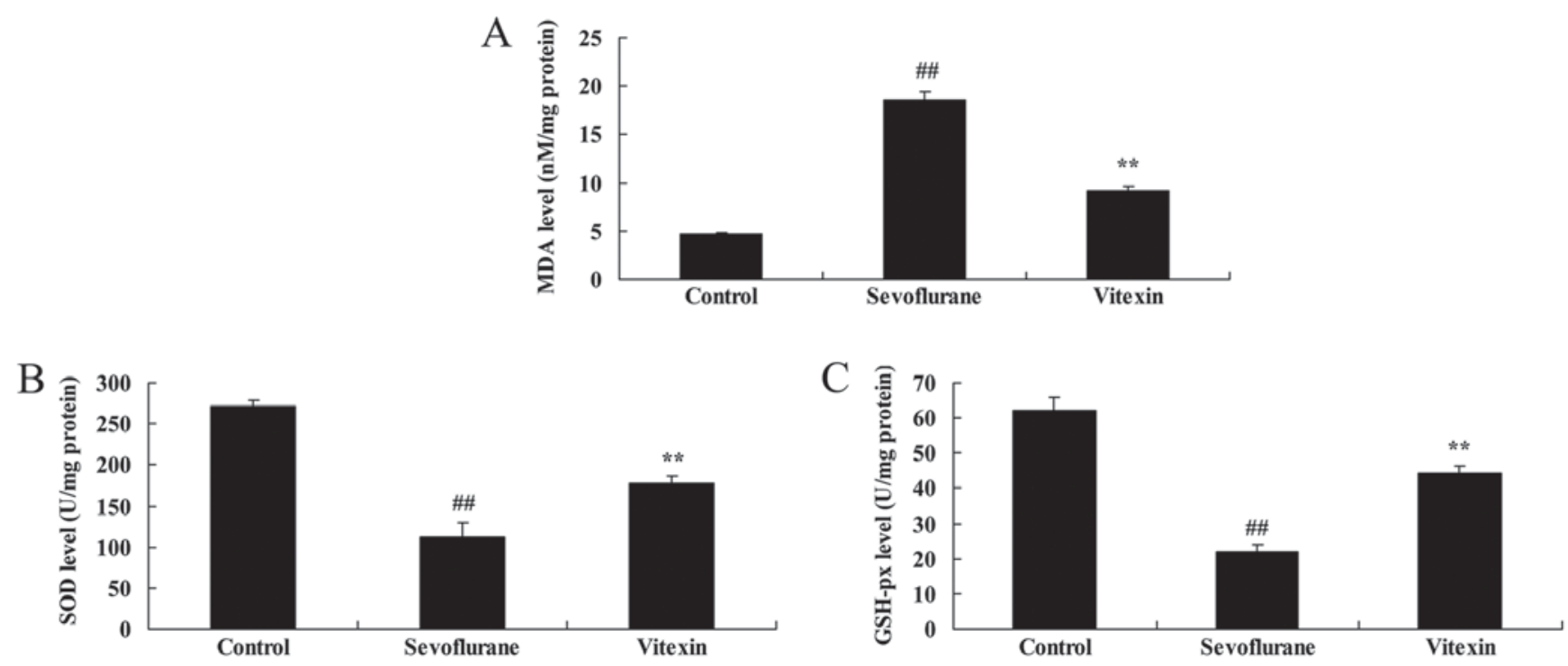

Figure 3. The effects of Vitexin on MDA, SOD and GSH-px activities of sevoflurane-induced newborn rat. The effects of Vitexin on (A) MDA, (B) SOD and (C) GSH-px activities of sevoflurane-induced newborn rat. Control, control group; Sevoflurane, sevoflurane model group; Vitexin, Vitexin + sevoflurane model group. ${ }^{* *} \mathrm{P}<0.01$ compared with the sevoflurane model group; ${ }^{\# \#} \mathrm{P}<0.01$ compared with the control group.

suppressed HIF-1 $\alpha$, VEGF and p38 MAPK expression, and promoted VEGF protein expression in sevoflurane-induced newborn rat (Fig. 4).

The effects of Vitexin on cell growth of sevoflurane-induced human neuroglioma cells. Then, we needed to explore the effects of Vitexin on cell growth of sevoflurane-induced human neuroglioma cells in vitro model, so H4 cell was gassed with $4.1 \%$ sevoflurane for $15 \mathrm{~min}$, and then treated with $100 \mu \mathrm{M}$ vitexin for $24 \mathrm{~h}$. These results showed that cell proliferation of sevoflurane-induced human neuroglioma cells was effectively inhibited, compared with control group (Fig. 5). Treatment with Vitexin increased the cell proliferation of sevoflurane-induced human neuroglioma cells, compared with sevoflurane-induced human neuroglioma cells group (Fig. 5).

The effects of Vitexin on caspase-3 activity and Bax protein expression of sevoflurane-induced human neuroglioma cells. We investigated the effects of Vitexin on nerve apoptosis of sevoflurane-induced human neuroglioma cells. There were significant increases of caspase- 3 activity and Bax protein expression of sevoflurane-induced human neuroglioma cells, compared with control group (Fig. 6). Treatment with Vitexin significantly inhibited caspase-3 activity and Bax protein expression of sevoflurane-induced human neuroglioma cells, compared with sevoflurane-induced human neuroglioma cells group (Fig. 6).

The effects of Vitexin on MDA, SOD and GSH-px activities of sevoflurane-induced human neuroglioma cells. To further investigate whether the effects of Vitexin on oxidative stress of sevoflurane-induced human neuroglioma cells, MDA, SOD and GSH-px activities were measured using ELISA KITS. As showed in Fig. 7, the increase of MDA level and inhibition of SOD and GSH-px levels were effectively in sevoflurane-induced human neuroglioma cells, compared with control group. Treatment with Vitexin significantly inhibited MDA level and increased SOD and GSH-px levels in sevoflurane-induced human neuroglioma cells (Fig. 7).

The effects of Vitexin on HIF-1 $\alpha, V E G F$ and p38 MAPK protein expression of sevoflurane-induced human neuroglioma cells. We performed western blot analysis to measure HIF-1 $\alpha$, VEGF and p38 MAPK protein expression in sevoflurane-induced human neuroglioma cells. As showed in Fig. 8, p38 MAPK protein expression were effectively increased, HIF-1 $\alpha$, and VEGF protein expression was effectively decreased in sevoflurane-induced human neuroglioma cells, 

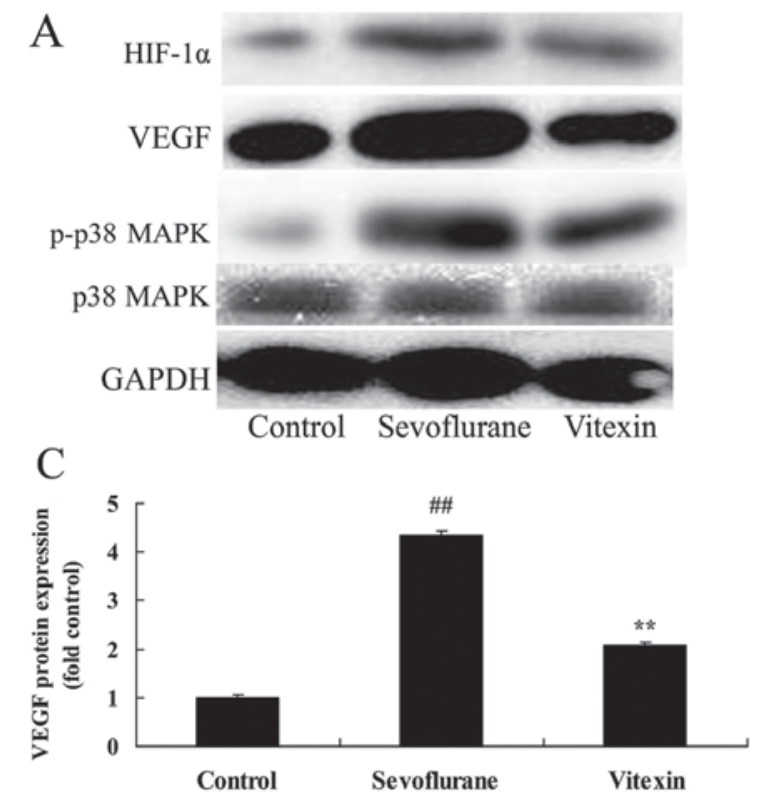
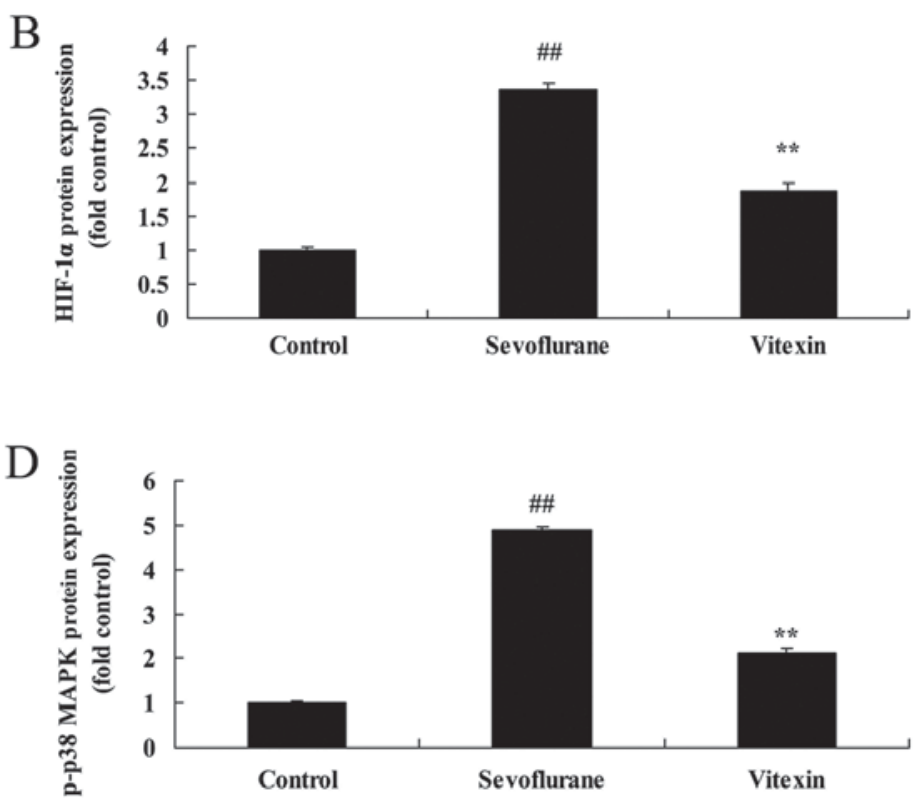

Figure 4. The effects of Vitexin on HIF-1 $\alpha$, VEGF and p38 MAPK protein expression of sevoflurane-induced newborn rat. The effects of Vitexin on (A) HIF-1 $\alpha$, VEGF and p38 MAPK protein expression and (B-D) statistical analysis of sevoflurane-induced newborn rat. Control, control group; Sevoflurane, sevoflurane model group; Vitexin, Vitexin + sevoflurane model group. ${ }^{* *} \mathrm{P}<0.01$ compared with the sevoflurane model group; ${ }^{\# \#} \mathrm{P}<0.01$ compared with the control group.

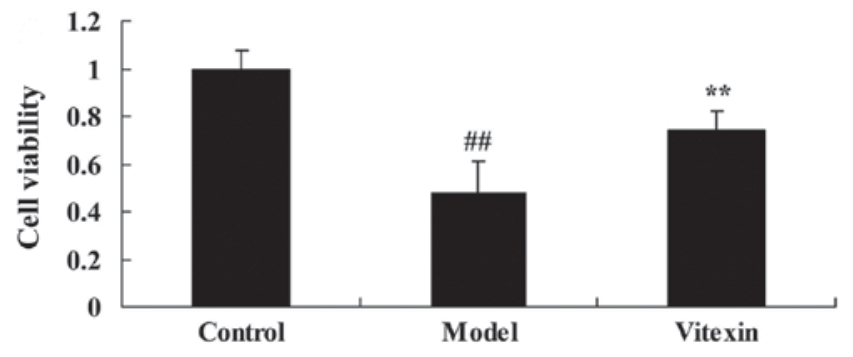

Figure 5. The effects of Vitexin on cell growth of sevoflurane-induced human neuroglioma cells. Control, control group; Model, sevoflurane model group; Vitexin, Vitexin + sevoflurane model group. ${ }^{* *} \mathrm{P}<0.01$ compared with the sevoflurane model group; ${ }^{\# \#} \mathrm{P}<0.01$ compared with the control group.

compared control group. However, treatment with Vitexin significantly suppressed HIF-1 $\alpha$ and p-p38 MAPK expression and induced VEGF protein expression in sevoflurane-induced human neuroglioma cells (Fig. 8).

\section{Discussion}

However, as we all know, the acting mechanisms of both intravenous anesthetic and inhaled anesthetic have not been clear so far (11). As for such a drug in the central nervous system, the safety deserves more attention when it facilitates surgical operation at the same time, especially the problem whether young children's neurological development is adversely affected, which is becoming urgent for medical workers to solve (11). More and more studies have gradually confirmed that there is a certain degree of neurotoxicity in sevoflurane and other inhaled anesthetics, which have significant impact on the development of children's cognitive abilities (12). In the current study, we observed that treatment with vitexin increased the cell proliferation of sevoflurane-induced human neuroglioma cells. Treatment with Vitexin significantly inhibited MDA level and increased SOD and GSH-px levels in sevoflurane-induced newborn rat or human neuroglioma cells. Sun et al suggested that vitexin possesses cardioprotective action against DOX-induced cardiotoxicity through anti-oxidative effects (10).

Bax is a Bcl-2 superfamily. Bcl-2 is an oncogene isolated from follicular lymphoma, namely, B cell lymphoma/Leukernia-2 (B cell lymphoma/Leukernia-2). Bcl-2 family can be divided into two categories: One is to promote apoptosis, namely, Bax, Bcl-xs, Bad, Bak, etc.; the other is to inhibit cell apoptosis, namely, Bcl-2, Bcl-xl, Mcl-1, Bag-1, Ced-9 and some viral genes (13). Once the cells are induced by apoptotic factors, they can transduce to the mitochondria, form a transmembrane channel in the mitochondrial outer membrane by oligomerization, or open the PT pore of the mitochondria, leading to the release of apoptotic factors in the mitochondria, to activate caspases, thus leading to cell apoptosis (14). These findings indicated that vitexin suppressed Bax protein expression $n$ sevoflurane-induced newborn rat or human neuroglioma cells. Dong et al demonstrated that vitexin protects against myocardial ischemia/reperfusion injury through suppression of Bax expression (15).

Caspases can be spontaneously activated or activated by a similar cascade of specific enzymes (16). The cascade of hydrolysed proteins is similar to the 'waterfall effect' of coagulation factor activation (17). Recent studies have shown that caspase family plays an important role in ischemic brain injury, as the start and execution of mammalian apoptosis, in which caspase- 3 is the most important apoptotic protease caspasein the downstream of cascade 'waterfall', and plays a pivotal role in the apoptotic process initiated by a variety of factors (18). The present data demonstrated that vitexin inhibited caspase-3 activity in sevoflurane-induced newborn rat or human neuroglioma cells.

HIF-1 is an oxygen-sensitive transcriptional regulator, which regulates more than 70 downstream target genes, 

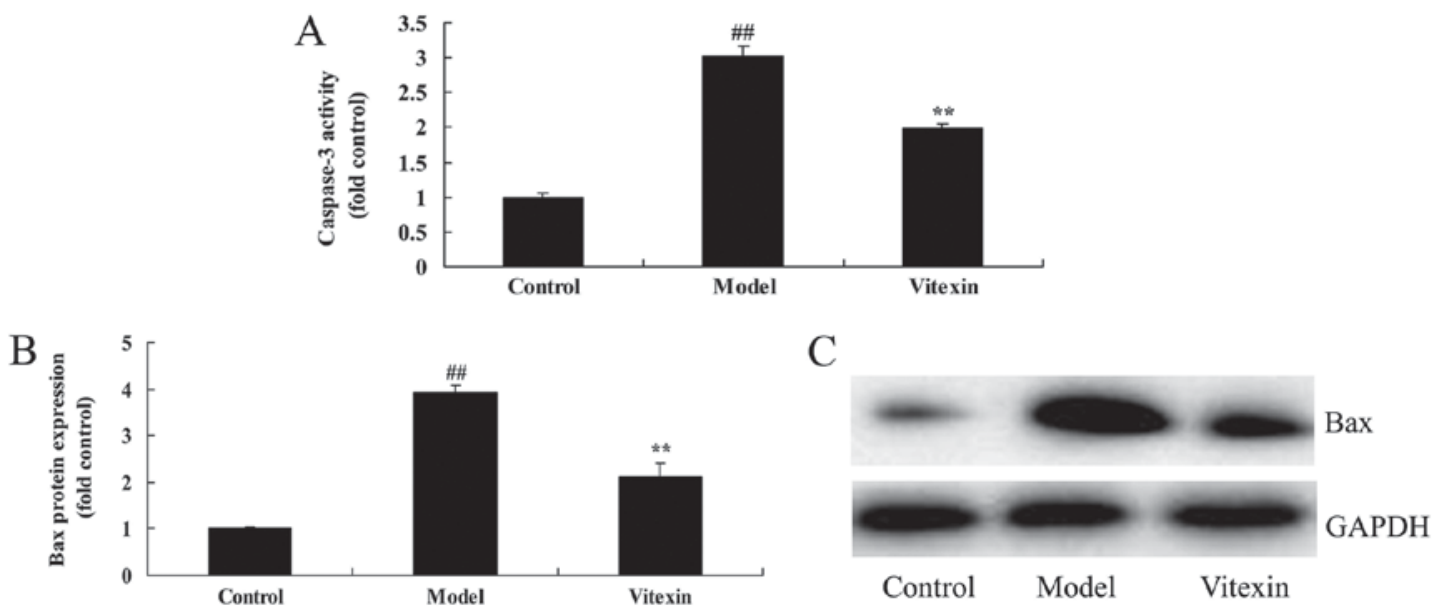

Figure 6. The effects of Vitexin on caspase-3 activity and Bax protein expression of sevoflurane-induced human neuroglioma cells. The effects of Vitexin (A) on caspase-3 activity and (B) Bax protein expression, and (C) statistical analysis of sevoflurane-induced human neuroglioma cells. Control, control group; Model, sevoflurane model group; Vitexin, Vitexin + sevoflurane model group. ${ }^{* *} \mathrm{P}<0.01$ compared with the sevoflurane model group; ${ }^{\# \#} \mathrm{P}<0.01$ compared with the control group.

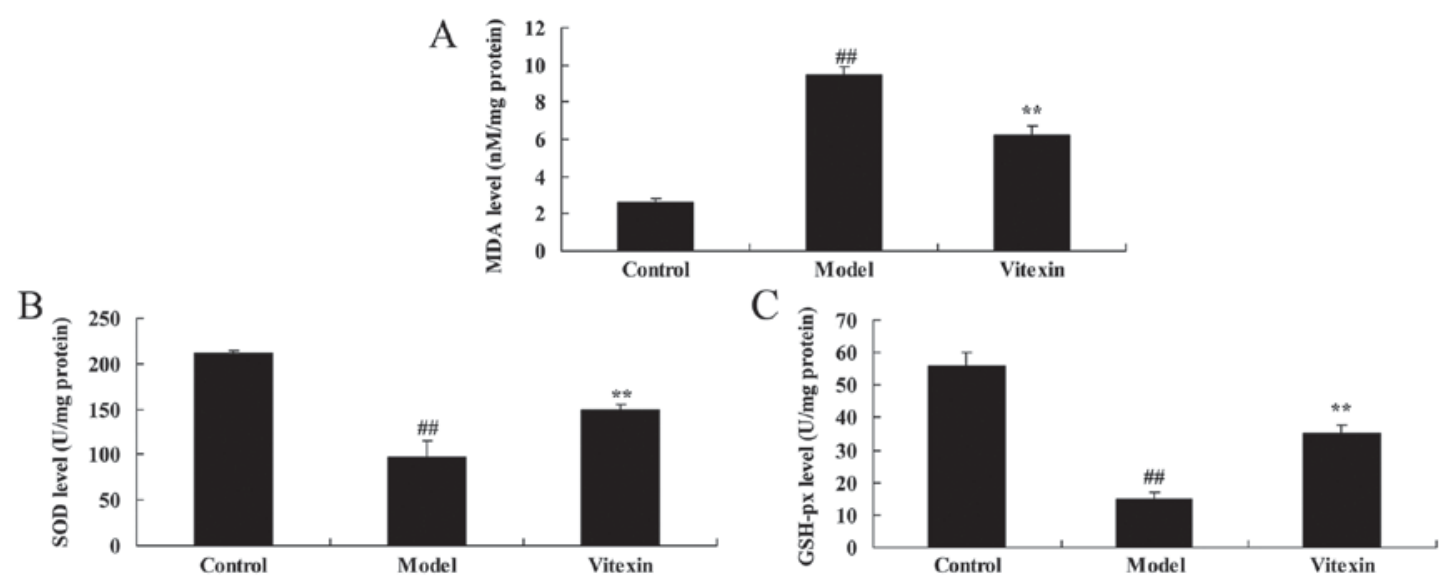

Figure 7. The effects of Vitexin on MDA, SOD and GSH-px activities of sevoflurane-induced human neuroglioma cells. The effects of Vitexin on (A) MDA, (B) SOD and (C) GSH-px activities of sevoflurane-induced human neuroglioma cells. Control, control group; Model, sevoflurane model group; Vitexin, Vitexin + sevoflurane model group. ${ }^{* *} \mathrm{P}<0.01$ compared with the sevoflurane model group; ${ }^{\# \# ~} \mathrm{P}<0.01$ compared with the control group.

A
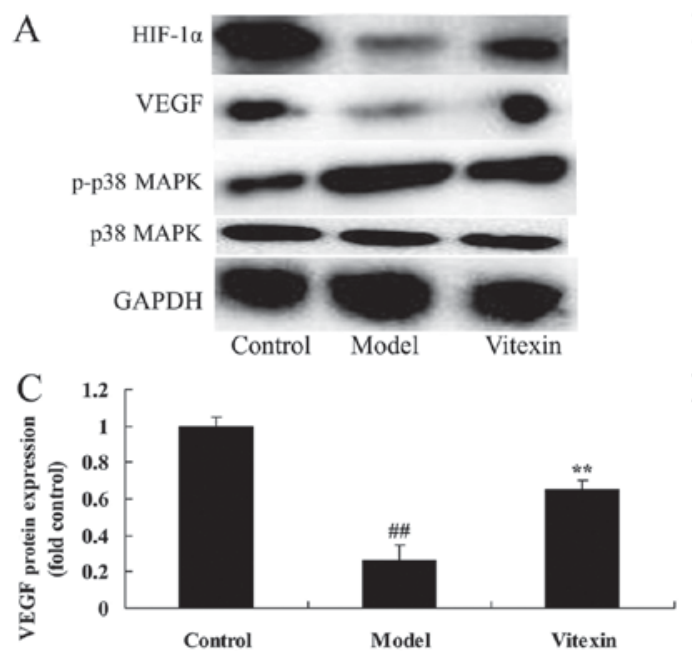

$\mathrm{B}$

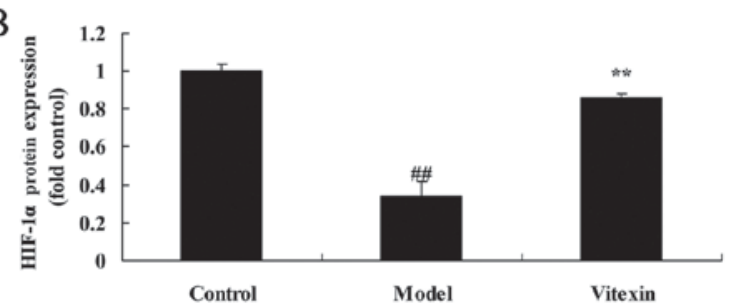

D

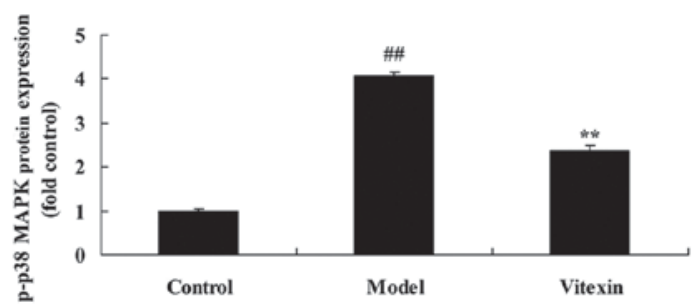

Figure 8. The effects of Vitexin on HIF-1 $\alpha$, VEGF and p38 MAPK protein expression of sevoflurane-induced human neuroglioma cells. The effects of Vitexin on (A) HIF-1 $\alpha$, VEGF and p38 MAPK protein expression and (B-D) statistical analysis of sevoflurane-induced human neuroglioma cells. Control, control group; Model, sevoflurane model group; Vitexin, Vitexin + sevoflurane model group. ${ }^{* * *} \mathrm{P}<0.01$ compared with the sevoflurane model group; ${ }^{\# \#} \mathrm{P}<0.01$ compared with the control group. 
including VEGF, EPO and GLUT (12). Both of them play an important role in the protection of cerebral ischemic injury. HIF-1 has two subunits: HIF-1 and HIF-1 $\beta$ (6). Some studies have shown that the upregulation of the expression of HIF-1 can play a neuroprotective role in the focal cerebral ischemic injury model, but a few studies suggest that its elevated expression may have some adverse effects (6). The results of the present study demonstrated that the protein of HIF-1 $\alpha$ expression in sevoflurane-induced newborn rat or human neuroglioma cells was induced by vitexin. The function and implication of HIF-1 $\alpha$ was behaved in hypoxi, however, the implication of HIF-1 $\alpha$ is limited value in normoxic conditions in sevoflurane-induced newborn rat or human neuroglioma cells. We need to be investigated in hypoxic and normoxic conditions for the further study.

It is believed that VEGF has a direct role in neurotrophy and protection in addition to the promoting effect on the growth of new blood vessels (19). Recently, it has been found that VEGF can protect injured neurons and promote vascular repair and nerve regeneration in injured spinal cord tissue by acting on the receptor, rapidly inducing nerve cell self-rescue, promoting nerve axon regeneration and Schwann cell growth, etc (20). VEGF can also promote the proliferation of spinal glial cells, especially astrocytes, and secrete glial cell line-derived neurotrophic factor through VEGFR-2 receptor (20). GDNF can prevent neuronal apoptosis and promote axon growth, thus indirectly protecting nerve cells (21). In the present study, vitexin induced VEGF protein expression in sevoflurane-induced newborn rat or human neuroglioma cells. Min et al indicated that vitexin reduces hypoxia-ischemia neonatal brain injury through suppression of HIF-1 $\alpha$ and VEGF expression (22). Choi et al reported that Vitexin has anti-metastatic potential in $\mathrm{PC} 12$ cells through HIF-1alpha inhibitor and VEGF expression (23). These results suggest that vitexin suppressed HIF-1 $\alpha$ and VEGF expression to prevent sevoflurane-induced neuronal apoptosis.

P38 mitogen-activated protein kinase (MAPK) signaling pathway is involved in the late apoptosis of a large number of neurons following ischemic brain injury. It is one of the important signal transduction pathways of apoptosis induced by ischemia reperfusion injury (8). p-p38 MAPK activates the transcription factorsin downstream and caspase family members (Caspase-3, -6 and -7) (24). In recent years, researchers use the TUNEL method to label DNA fragments and have confirmed that the hypoxia-ischemia in cultured neurons and in vivo can lead to neuronal apoptosis (16). The present findings indicated that vitexin suppressed p-p-38 MAPK protein expression in sevoflurane-induced newborn rat or human neuroglioma cells. Wang et al showed that vitexin alleviates LPS-induced islet cell injury by inhibiting of p38 signaling pathways (25).

In conclusion, this study indicated that the protective effect of vitexin reduces sevoflurane-induced neuronal apoptosis through HIF-1 $\alpha$, VEGF and p38 MAPK signaling pathway in newborn rat or human cell. In this study, we used newborn rat model and human neuroglioma $\mathrm{H} 4$ cells model, which showed that the protective effect of vitexin reduces neuronal apoptosis in vitro and in newborn rats by sevoflurane. Furthermore, our results suggest that vitexin protects sevoflurane-induced neuronal apoptosis in brain, which may be assisted adverse reactions during anesthetic in clinical application.

\section{References}

1. Liu X, Song X, Yuan T, He J, Wang X and Wang Q: Effects of calpain on sevoflurane-induced aged rats hippocampal neuronal apoptosis. Aging Clin Exp Res 28: 633-639, 2016.

2. Istaphanous GK, Howard J, Nan X, Hughes EA, McCann JC, McAuliffe JJ, Danzer SC and Loepke AW: Comparison of the neuroapoptotic properties of equipotent anesthetic concentrations of desflurane, isoflurane, or sevoflurane in neonatal mice. Anesthesiology 114: 578-587, 2011.

3. Zhang DX, Zhang LM, Zhao XC and Sun W: Neuroprotective effects of erythropoietin against sevoflurane-induced neuronal apoptosis in primary rat cortical neurons involving the EPOR-Erk1/2-Nrf2/Bach1 signal pathway. Biomed Pharmacother 87: 332-341, 2017.

4. Wang LY, Tang ZJ and Han YZ: Neuroprotective effects of caffeic acid phenethyl ester against sevofluraneinduced neuronal degeneration in the hippocampus of neonatal rats involve MAPK and PI3K/Akt signaling pathways. Mol Med Rep 14: 3403-3412, 2016.

5. Bellanti F, Mirabella L, Mitarotonda D, Blonda M, Tamborra R, Cinnella G, Fersini A, Ambrosi A, Dambrosio M, Vendemiale G and Serviddio G: Propofol but not sevoflurane prevents mitochondrial dysfunction and oxidative stress by limiting HIF-1 $\alpha$ activation in hepatic ischemia/reperfusion injury. Free Radic Biol Med 96: 323-333, 2016.

6. Ye Z, Guo Q, Xia P, Wang N, Wang E and Yuan Y: Sevoflurane postconditioning involves an up-regulation of HIF- $1 \alpha$ and HO-1 expression via PI3K/Akt pathway in a rat model of focal cerebral ischemia. Brain Res 1463: 63-74, 2012.

7. Aminzadeh A, Dehpour AR, Safa M, Mirzamohammadi S and Sharifi AM: Investigating the protective effect of lithium against high glucose-induced neurotoxicity in PC12 cells: Involvements of ROS, JNK and P38 MAPKs, and apoptotic mitochondria pathway. Cell Mol Neurobiol 34: 1143-1150, 2014.

8. Ghasemi R, Zarifkar A, Rastegar K, Maghsoudi N and Moosavi M: Insulin protects against A $\beta$-induced spatial memory impairment, hippocampal apoptosis and MAPKs signaling disruption. Neuropharmacology 85: 113-120, 2014.

9. Che X, Wang X, Zhang J, Peng C, Zhen Y, Shao X, Zhang G and Dong L: Vitexin exerts cardioprotective effect on chronic myocardial ischemia/reperfusion injury in rats via inhibiting myocardial apoptosis and lipid peroxidation. Am J Transl Res 8: 3319-3328, 2016 .

10. Sun Z, Yan B, Yu WY, Yao X, Ma X, Sheng G and Ma Q: Vitexin attenuates acute doxorubicin cardiotoxicity in rats via the suppression of oxidative stress, inflammation and apoptosis and the activation of FOXO3a. Exp Ther Med 12: 1879-1884, 2016.

11. Zhang LM, Zhao XC, Sun WB, Li R and Jiang XJ: Sevoflurane post-conditioning protects primary rat cortical neurons against oxygen-glucose deprivation/resuscitation via down-regulation in mitochondrial apoptosis axis of Bid, Bim, Puma-Bax and Bak mediated by Erk1/2. J Neurol Sci 357: 80-87, 2015

12. Liu XL, Lu J and Xing J: Stabilization of HIF-1 $\alpha$ modulates VEGF and Caspase-3 in the hippocampus of rats following transient global ischemia induced by asphyxial cardiac arrest. Life Sci 151: 243-249, 2016.

13. Zuo D, Lin L, Liu Y, Wang C, Xu J, Sun F, Li L, Li Z and Wu Y: Baicalin attenuates ketamine-induced neurotoxicity in the developing rats: Involvement of PI3K/Akt and CREB/BDNF/Bcl-2 pathways. Neurotox Res 30: 159-172, 2016.

14. Nam YJ, Kim A, Lee MS, Shin YK, Sohn DS and Lee CS: Lamotrigine attenuates proteasome inhibition-induced apoptosis by suppressing the activation of the mitochondrial pathway and the caspase-8- and bid-dependent pathways. Neurochem Res 41: 2503-2516, 2016.

15. Dong L, Fan Y, Shao X and Chen Z: Vitexin protects against myocardial ischemia/reperfusion injury in Langendorff-perfused rat hearts by attenuating inflammatory response and apoptosis. Food Chem Toxicol 49: 3211-3216, 2011.

16. So EC, Lin YX, Tseng CH, Pan BS, Cheng KS, Wong KL, Hao LJ, Wang YK and Huang BM: Midazolam induces apoptosis in MA-10 mouse Leydig tumor cells through caspase activation and the involvement of MAPK signaling pathway. Onco Targets Ther 7: 211-221, 2014. 
17. Liu HC, Zhang Y, Zhang S, Xin T, Li WH, Wu WL, Pang Q and Chen YZ: Correlation research on the protein expression (p75NTR, bax, bcl-2, and caspase-3) and cortical neuron apoptosis following mechanical injury in rat. Eur Rev Med Pharmacol Sci 19: 3459-3467, 2015.

18. Chen B, Wang G, Li W, Liu W, Lin R, Tao J, Jiang M, Chen L and Wang Y: Memantine attenuates cell apoptosis by suppressing the calpain-caspase-3 pathway in an experimental model of ischemic stroke. Exp Cell Res 351: 163-172, 2017.

19. Cui W, Li W, Han R, Mak S, Zhang H, Hu S, Rong J and Han Y: PI3-K/Akt and ERK pathways activated by VEGF play opposite roles in $\mathrm{MPP}^{+}$-induced neuronal apoptosis. Neurochem Int 59: 945-953, 2011

20. Cui W, Li W, Zhao Y, Mak S, Gao Y, Luo J, Zhang H, Liu Y, Carlier PR, Rong J and Han Y: Preventing $\mathrm{H}_{2} \mathrm{O}_{2}$-induced apoptosis in cerebellar granule neurons by regulating the VEGFR-2/Akt signaling pathway using a novel dimeric antiacetylcholinesterase bis(12)-hupyridone. Brain Res 1394: 14-23, 2011.

21. Rong W, Wang J, Liu X, Jiang L, Wei F, Hu X, Han X and Liu Z: Naringin treatment improves functional recovery by increasing BDNF and VEGF expression, inhibiting neuronal apoptosis after spinal cord injury. Neurochem Res 37: 1615-1623, 2012.
22. Min JW, Hu JJ, He M, Sanchez RM, Huang WX, Liu YQ, Bsoul NB, Han S, Yin J, Liu WH, et al: Vitexin reduces hypoxia-ischemia neonatal brain injury by the inhibition of HIF-1alpha in a rat pup model. Neuropharmacology 99: 38-50, 2015.

23. Choi HJ, Eun JS, Kim BG, Kim SY, Jeon H and Soh Y: Vitexin, an HIF-1alpha inhibitor, has anti-metastatic potential in PC12 cells. Mol Cells 22: 291-299, 2006.

24. Kwon SH, Kim JA, Hong SI, Jung YH, Kim HC, Lee SY and Jang CG: Loganin protects against hydrogen peroxide-induced apoptosis by inhibiting phosphorylation of JNK, p38, and ERK 1/2 MAPKs in SH-SY5Y cells. Neurochem Int 58: 533-541, 2011.

25. Wang F, Yin J, Ma Y, Jiang H and Li Y: Vitexin alleviates lipopolysaccharide-induced islet cell injury by inhibiting HMGB1 release. Mol Med Rep 15: 1079-1086, 2017. 